\title{
Governança de dados aplicada ao planejamento urbano municipal
}

\author{
Bianca da Rocha Bartolomei, Melise Maria Veiga de Paula, \\ Vanessa Cristina Oliveira de Souza \\ ${ }^{1}$ Instituto de engenharia de sistemas e tecnologia da informação - \\ Universidade Federal de Itajubá (UNIFEI) \\ Caixa Postal 50 - 37.500-903 - Itajubá - MG - Brazil \\ \{biancabartolomei, melise, vanessasouza\}@unifei.edu.br
}

\begin{abstract}
With the growth of cities, greater investments are required in urban planning, the objective of which is to guide actions of municipal public management in order to minimize urban problems and improve the quality of life of citizens. The organization and analysis of data about a municipality can help this process insofar as it allows for more assertive decisions. The data in question can be from different sources, structured or not, sensitive and also have inconsistencies. In order to solve this problem, the proposed solution for this work is the elaboration and development of a model capable of handling heterogeneous data, collecting, integrating and making them available for decision making by stakeholders in order to assist municipal urban planning.
\end{abstract}

Resumo. Com crescimento das cidades, são exigidos maiores investimentos em planejamento urbano, cujo objetivo é orientar ações da gestão pública municipal a fim de minimizar os problemas urbanos e melhorar a qualidade de vida dos cidadãos. A organização e análise de dados sobre um município pode ajudar esse processo na medida em que possibilita decisões mais assertivas. Os dados em questão podem ser de diferentes fontes, estruturados ou não, sensíveis $e$, ainda, apresentar inconsistências. Com o intuito de solucionar esse problema, a proposta de solução deste trabalho é a elaboração e o desenvolvimento de um modelo capaz de lidar com dados heterogêneos, coletá-los, integrá-los e disponibilizá-los para a tomada de decisão pelos stakeholders a fim de auxiliar o planejamento urbano municipal.

\section{Introdução}

As Nações Unidas estimam que cerca de $66 \%$ da população mundial viverão em áreas urbanas até 2050 [Ahvenniemi et al. 2017]. Caso aconteça de forma desordenada, a urbanização pode gerar diferentes impactos negativos, como a formação de ilhas de calor e aumento da violência. Nesse sentido, é necessário o planejamento urbano municipal para estruturar e organizar as cidades, uma vez que permite a correta alocação de recursos, a mobilização de setores da sociedade para a resolução de problemas coletivos e a criação de metas e ações para a gestão pública. Para a elaboração do planejamento urbano municipal, é preciso conhecer o município e sua organização territorial e identificar suas potencialidades, problemas e demandas, assim como ter uma visão sistematizada de aspectos sociais, políticos e econômicos [Fritz et al. 2020]. A utilização de dados provenientes 
de fontes variadas pode fornecer informações importantes sobre um município, auxiliando seu planejamento urbano e a tomada de decisões mais assertivas pelos gestores.

Existem estudos e projetos que propõem melhorias e soluções apoiadas no uso de tecnologias de informação e comunicação (TICs) para os problemas urbanos. Por exemplo, em [Psyllidis et al. 2015], é proposto um framework para o desenvolvimento de um sistema que dê suporte à tomada de decisão em um município a partir de dados heterogêneos usando métodos de ciência dos dados, integração semântica e crowdsourcing. Já em [Lupi 2019], foi elaborado um modelo para a gerenciar a produção e a obtenção de dados sobre o município e sua utilização pelos stakeholders locais a partir da governança de dados.

O uso de TICs em soluções de planejamento urbano é um desafio, uma vez que problemas como a falta de infraestrutura tecnológica adequada, o analfabetismo tecnológico e a dificuldade de acesso aos dados podem inviabilizar seu desenvolvimento [Tan and Taeihagh 2020]. Os dados podem ser obtidos a partir de fontes como imagens de sensoriamento remoto e redes sociais [Kong et al. 2020], mas também de processos e planos administrativos municipais que são realizados de forma independente entre si.

Dessa forma, o verdadeiro problema não é a inexistência de dados sobre um município, mas sim a heterogeneidade desses dados, uma vez que podem ser disponibilizados de forma descentralizada por fontes independentes e possuir organizações diferentes, ou, ainda, conter inconsistências ou informações sensíveis [Lupi 2019]. Portanto, o objetivo desse trabalho é o desenvolvimento de uma solução capaz de lidar com dados heterogêneos, integrá-los e disponibilizá-los a partir de um processo que garanta a qualidade, a segurança e o valor das informações a fim de apoiar a tomada de decisões no planejamento urbano municipal.

\section{Projeto de Pesquisa}

Segundo [Janssen et al. 2020], a governança de dados pode ser entendida como uma maneira de definir, aplicar e monitorar regras para o correto funcionamento dos dados e processos dentro e entre organizações. A governança de dados é uma das formas de definir a integridade, consistência, segurança e qualidade de dados e de processos. Por essa razão, a proposta de solução a ser desenvolvida para o problema de pesquisa seguirá essa abordagem, definindo-se sete etapas principais: definir os stakeholders; contextualizar e entender o problema; definir temas dos dados; elencar critérios de qualidade e segurança; coletar diferentes tipos de dados; integrar os dados; e disponibilizar os dados ou informações.

A primeira etapa é a definição de quem são os stakeholders e de que forma eles podem ser beneficiados com a exploração controlada dos dados. Entende-se que stakeholders são todos os interessados nos dados sobre a dinâmica do município, mesmo que o principal seja a própria administração pública. Entre alguns exemplos, destacam-se os cidadãos, empresas e órgãos públicos, como a polícia civil. O desenvolvimento da solução computacional proposta será restrita para apenas um grupo de stakeholders.

A segunda etapa consiste em entender o contexto do problema segundo a perspectiva do grupo de stakeholders escolhido. Para isso, busca-se avaliar os impactos da falta de informação consistente e integrada no contexto do planejamento urbano para esses stakeholders.

A terceira etapa é a definição de tema, ou seja, é necessário estabelecer uma es- 
tratégia de agrupamento dos dados em assuntos pertinentes ao planejamento do município. Por exemplo, áreas de saúde e de transporte. Nesse sentido, consideram-se os resultados das etapas anteriores. Algumas das possíveis estratégias seriam entender como os recursos municipais são divididos ou então adotar um padrão, como por exemplo as dimensões de smart cities [Ismagilova et al. 2019].

Os critérios de qualidade e segurança para os dados e os algoritmos serão baseados em trabalhos mais recentes na área de governança de dados, que serão elencados a partir da revisão da literatura sobre o assunto, observando o atendimento às diretrizes da Lei Geral de Proteção de Dados (LGPD) [Brasil 2018].

A quinta etapa é a captura dos diferentes tipos de dados. O primeiro passo é conhecer os dados disponíveis, então classificá-los e avaliá-los para identificar quais os tratamentos necessários para cada conjunto. Isso porque os dados podem ser estruturados, semi-estruturados ou não-estruturados. Além disso, é importante que os dados cumpram os critérios definidos na etapa anterior.

A etapa de integração de dados considera as etapas de captura de dados, definição de stakeholders e definição de temas, já que será responsável por integrar os dados em um tema específico segundo o interesse dos stakeholders selecionados e a conveniência com os critérios de governança de dados adotados. A integração pode ser realizada a partir de algoritmos de mineração de texto ou dados, aprendizado de máquina, processamento de linguagem natural ou métodos estatísticos, conforme o mais adequado para os conjuntos de dados disponíveis.

A disponibilização de dados é uma etapa importante, já que é a entrega dos dados aos stakeholders escolhidos, devendo também cumprir requisitos de qualidade. A entrega pode ser feita por meio de APIs, de forma a dar uma margem de liberdade ao uso do dado, ou, ainda, por visualização de informações, em que existe já um grau de interpretação das potencialidades existentes no sistema.

\section{Projeto de Avaliação}

A execução dessa pesquisa considera os projetos que são realizados pelo Núcleo Estratégico Interdisciplinar de Resiliência Urbana (NEIRU) da Universidade Federal de Itajubá. O NEIRU é um grupo de pesquisa e extensão que executa serviços que apoiam a gestão pública de municípios do Sul de Minas Gerais. Dentre eles, pode-se destacar: elaboração e revisão de planos diretores, planos de saneamento básico, planos de meio ambiente e atualização do cadastro multifinalitário de imóveis. A execução de cada um desses planos gera diferentes produtos finais para o município. No caso dos planos diretores, por exemplo, a minuta de lei sobre uso e ocupação do solo é um exemplo. Além dos produtos finais, são gerados outros produtos e dados intermediários como, ainda no exemplo do plano diretor, mapas, dados coletados através de pesquisa de opinião e outros tipos de dados que podem auxiliar processos administrativos.

Propõe-se um estudo de caso para a avaliação da solução, pois essa técnica possibilita uma análise qualitativa [Wohlin et al. 2012], que faz sentido em um contexto complexo que é a dinâmica de um município, seus stakeholders e seus interesses. Assim, tomariase um município, ao qual o NEIRU presta serviços, como objeto de estudo. Além disso, pretende-se também a aplicação de questionários e entrevistas nos stakeholders identificados com o objetivo de serem obtidas avaliações mais detalhadas da solução do problema. 


\section{Atividades realizadas}

As primeiras atividades que estão sendo realizadas são: a revisão bibliográfica e o levantamento dos conjuntos de dados disponíveis. A revisão bibliográfica busca entender o contexto do problema, ou seja, o planejamento urbano de um município e identificar o que tem sido feito de mais recente na área de governança de dados a fim de assegurar a consistência, a qualidade, o valor e a segurança dos dados e processos. Também, têm-se buscado como os diferentes tipos de dados podem ser integrados, quais são as técnicas disponíveis e as mais adequadas. A segunda atividade é o levantamento das fontes de dados disponíveis para as cidades atendidas pelo NEIRU, com especial atenção para os planos desenvolvidos, explicados a seguir.

O Plano Diretor Municipal (PDM) é definido pela Constituição Federal e pelo Estatuto da Cidade como o instrumento básico para organização, desenvolvimento e expansão do município. O PDM é obrigatório para municípios com mais de 20 mil habitantes, deve ser aprovado pela Câmara Municipal e revisado, no mínimo, a cada dez anos. Tudo o que for resultante do PDM, como os documentos e os resultados, deve ser público e acessível a qualquer interessado [Brasil 1988]. No Estatuto da Cidade é definido que é competência da União, Estados, Distrito Federal e Municípios a promoção de condições habitacionais e de saneamento básico. Nesse sentido, a Lei $\mathrm{n}^{\circ} 11.445$ diz que a titularidade dos serviços de saneamento básico são dos municípios e que o titular deve elaborar os planos de saneamento básico, justificando a elaboração e revisão do Plano Municipal de Saneamento Básico (PMSB) [Brasil 2007]. O Plano Municipal de Meio Ambiente (PMMA) é definido pela Política Nacional do Meio Ambiente, cujo objetivo é a preservação, recuperação e melhoria ambiental, de forma a dar condições ao desenvolvimento sócio-econômico, como definido na Lei ${ }^{\circ} 6.938$ [Brasil 1981]. O Cadastro Imobiliário tem por objetivo o cadastro dos imóveis do município, seja edificado ou não. Esse cadastro, apesar de poder ser usado para finalidades diferentes, tem como um dos principais objetivos a incidência de impostos sobre a área urbana, como por exemplo o Imposto Predial e Territorial Urbano (IPTU), como define o Decreto-lei no 82 [Brasil 1966].

\section{Considerações Finais}

A crescente urbanização é um dos desafios importantes dos próximos anos para as gestões públicas dos municípios, por essa razão é importante conhecer a organização do território municipal, entender a dinâmica social, política e econômica, e identificar as potencialidades, desafios e problemas do município. Dessa forma, viabilizar a eficácia e a eficiência do planejamento urbano municipal é importante. Para isso, uma das possibilidades é a descoberta de conhecimento por meio da integração de dados, em que destacam-se os provenientes de processos municipais que acontecem naturalmente em virtude de leis que determinam as competências dos municípios.

Portanto, esse trabalho propõe a coleta e integração de dados sobre um município, e posterior descoberta de conhecimento a partir de uma abordagem de governança de dados a fim de garantir a qualidade, segurança, consistência e valor dos dados e processos. Pretende-se também garantir a entrega dos dados e informações descobertas aos stakeholders selecionados. É definida como forma de avaliação do projeto o estudo de caso de um município, avaliando de forma qualitativa os impactos da solução desenvolvida para os stakeholders locais identificados. 


\section{Referências}

Ahvenniemi, H., Huovila, A., Pinto-Seppä, I., and Airaksinen, M. (2017). What are the differences between sustainable and smart cities? Cities, 60:234-245.

Brasil (1966). Decreto-lei no 82, de 26 de dezembro de 1966. [Online; acessado em 21 de Fevereiro de 2021].

Brasil (1981). Lei no 6.938, de 31 de agosto de 1981. [Online; acessado em 21 de Fevereiro de 2021].

Brasil (1988). Constituição da República Federativa do Brasil. Centro Gráfico, Brasília, DF.

Brasil (2007). Lei n ${ }^{\circ} 11.445$, de 5 de janeiro de 2007. [Online; acessado em 21 de Fevereiro de 2021].

Brasil (2018). Lei no 13.709, de 14 de agosto de 2018. [Online; acessado em 20 de Março de 2021].

Fritz, R. T., Pfeiffer, C. R., and de Pina Filho, A. C. (2020). A contribuição da engenharia urbana na solução de problemas territoriais. Espaço Urbano Volume, page 49.

Ismagilova, E., Hughes, L., Dwivedi, Y. K., and Raman, K. R. (2019). Smart cities: Advances in research - an information systems perspective. International Journal of Information Management, 47:88-100.

Janssen, M., Brous, P., Estevez, E., Barbosa, L. S., and Janowski, T. (2020). Data governance: Organizing data for trustworthy artificial intelligence. Government Information Quarterly, 37(3):101493.

Kong, L., Liu, Z., and Wu, J. (2020). A systematic review of big data-based urban sustainability research: State-of-the-science and future directions. Journal of Cleaner Production, page 123142 .

Lupi, L. (2019). City data plan: The conceptualisation of a policy instrument for data governance in smart cities. Urban Science, 3(3):91.

Psyllidis, A., Bozzon, A., Bocconi, S., and Bolivar, C. T. (2015). A platform for urban analytics and semantic data integration in city planning. In International conference on computer-aided architectural design futures, pages 21-36. Springer.

Tan, S. Y. and Taeihagh, A. (2020). Smart city governance in developing countries: A systematic literature review. sustainability, 12(3):899.

Wohlin, C., Runeson, P., Höst, M., Ohlsson, M. C., Regnell, B., and Wesslén, A. (2012). Experimentation in software engineering. Springer Science \& Business Media. 